\title{
Delimitação e avaliação de reservas em jazigos aluvionares diamantíferos a partir da acumulação de teores
}

\author{
Delimitation and evaluation of reserves of diamond alluvial \\ deposits through grade accumulation
}

\section{José A. Almeida}

Doutorado em Engenharia de Minas, Professor do DCT e Investigador do CICEGe, FCT-Universidade Nova de Lisboa, Monte da Caparica, Portugal E-mail: ja@fct.unl.pt

\section{Resumo}

Em inúmeros depósitos minerais, a distribuição de teores na transição entre as várias formações geológicas presentes é, normalmente, abrupta ou até irregular. Nestes casos, a avaliação de reservas e a quantificação local de parâmetros de qualidade têm de tomar em conta a existência das heterogeneidades que condicionam as transições bruscas de teores da variável em estudo, no espaço. Nesse artigo é apresentada uma metodologia de avaliação de reservas por estimação geoestatística, que tem em conta as zonalidades espaciais resultantes da gênese dos jazigos e que se traduz por transições abruptas de teores entre as diversas formações geológicas. Embora o desenvolvimento dessa metodologia seja apresentado para duas fases geológicas, a generalização para uma situação multi-fase, do ponto de vista morfológico, é imediata. A metodologia proposta é ilustrada na avaliação de reservas de diamantes num jazigo aluvionar, envolvendo a estimação da possança e acumulação em duas unidades geológicas (teores superiores a zero e teores iguais a zero).

Palavras-chave: Avaliação de reservas, jazigo aluvionar de diamantes, krigagem, acumulação, possança.

\section{Abstract}

In several mineral deposits, the distribution of grades in the transitions between adjacent geologic units is normally sharp or heterogeneous, creating a strong zoning effect. In these cases, the evaluation of reserves and the local estimation of grades must take into account the existence of this zoning effect. Herein is presented a methodology for the geostatistical estimation of reserves, which takes into account the spatial zoning effect of the grades, and leads to sharp transitions between distinct geologic units. Although the development of this methodology is presented for two geologic phases, the generalization for a situation of more units, simultaneously, is straightforward. This methodology is illustrated in the evaluation of diamond reserves in an alluvial deposit, involving the estimation of accumulation and thickness within two distinct geologic units (diamond grades higher than zero and grades equals to zero).

Keywords: Reserves evaluation, alluvial diamond deposits, krigging, accumulation, thickness. 


\section{Introdução}

O problema clássico da geoestatística consiste na caracterização espacial de uma ou várias propriedades descritivas de um determinado sistema em estudo, condicionado a uma partição em vários subsistemas, de características mais homogêneas do que o sistema considerado como um todo (Matheron, 1970; Garcia-Pereira, 1994; Goovaerts, 1997; Chiles \& Delfiner, 1999).

Entre estes sistemas, incluem-se os depósitos minerais, onde as características petrofísicas e geoquímicas mudam bruscamente no contato entre as várias unidades envolvidas. Assim a avaliação geoestatística de reservas tem de ter em conta esse efeito de zonalidade (Almeida et al, 1997). No caso de não se introduzir, a priori, a informação sobre a diferenciação morfológica na estimação das propriedades, o resultado seria um mapa atenuado e, geologicamente, irrealista.

A abordagem clássica geoestatística realiza a modelação em duas etapas (Journel \& Isaaks, 1984; Soares, 1990):

i) Caracterização da forma dos vários subsistemas com o auxílio de variáveis descritoras da respectiva morfologia (possança, indicatriz, litologias, litoclasses, etc.)

ii) Caracterização das propriedades de interesse (teores, porosidade, índices, etc.) condicionadas à morfologia. Essa abordagem bietápica pode ter várias variantes, uma das quais é a caracterização da morfologia recorrendo-se a métodos clássicos de desenho geológico de perfis e à interpolação entre perfis, seguida da aplicação de metodologias geoestatísticas, para a geração de mapas das propriedades de interesse.

Esse procedimento é o mais utilizado hoje em dia e o que conduz, regra geral, aos melhores resultados. Todavia, nos casos onde existe escassez de informação, para um determinado subsistema geológico, o resultado pode ser um forte enviesamento (sub ou sobreestimação) na avaliação de reservas.

Nesse artigo, é apresentada uma metodologia geoestatística de estimação de reservas, cujo objectivo é o zonamento de massas minerais e a delimitação de áreas com características bem definidas do ponto de vista dos teores ou das propriedades que as definem.

A metodologia explanada é aplicada a um depósito aluvionar de diamantes. Nesses depósitos, a localização dos diamantes tende a aparecer concentrada em certos locais (armadilhas ou trapsites), evidenciando transições bruscas entre os locais com teores (quilates $/ \mathrm{m}^{3}$ ) superiores zero e os com teores nulos (áreas complementares). A gênese geológica das jazidas, relacionada com o transporte e com a deposição das pedras em armadilhas, pode gerar áreas mineralizadas diferenciadas espacialmente, segundo as dimensões das pedras, o que tem uma influência determinante na distribuição de teores (Marshalla \& Baxter-Brownb, 1995; Rombouts , 1995).

\section{Estimação simultânea da morfologia e dos teores baseada na acumulação dos teores}

\subsection{Definição das variáveis acumulação relativa $Y_{a i}(x)$ e possança relativa $Y_{p i}(x)$}

Embora essa metodologia possa ser generalizada para um conjunto de $N$ partições geológicas complementares $\left(X_{1}\right.$, $X_{2}, \ldots X_{N}$ ), consideram-se, para efeitos meramente ilustrativos, apenas duas formações geológicas, $X_{1}$ e $X_{2}$, em que as suas características internas, em termos de teores, são distintas e apresentam uma transição brusca bem definida, efeito zonal ou morfológico.

Nesse estudo, a metodologia de controlo zonal é apresentada com as variáveis acumulação $a(x)$ e espessura $p(x)$. Assim, em cada localização genérica $x$ da área $A, A=X_{1} \cup X_{2}$, consideram-se as seguintes medições:

$p(x)$ - possança da área mineralizada (m)

$z(x)$ - teor em diamantes (quilates $/ \mathrm{m}^{3}$ )

$a(x)=p(x) \cdot z(x)$ - acumulação da variável $z$ na localização $x$ (quilates $/ \mathrm{m}^{2}$ )

Considera-se, ainda, a codificação em várias unidades geológicas $X_{i}, i=1, N$, utilizando o vetor binário $K_{i}(x)$ - para codificar duas fases apenas é necessária uma variável indicatriz. Para $N$ fases, considera-se o vetor de variáveis binárias $\left.K_{i}(x), i=1, \ldots N\right)$ :

$K_{i}(x)=\left\{\begin{array}{l}1 \text { se } x \in X_{i} \\ 0 \text { se } x \in X_{j} \quad \mathrm{i} \neq \mathrm{j}\end{array}\right.$

Consideram-se, também, os dois seguintes vetores auxiliares, acumulação por unidade geológica

$Y_{a_{i}}(x)=a(x) \cdot K_{i}(x)=\left\{\begin{array}{l}a(x) \text { se } x \in X_{i} \\ 0 \text { se } x \notin X_{i}\end{array}\right.$

e possança de cada unidade geológica

$Y_{p_{i}}(x)=p(x) \cdot K_{i}(x)= \begin{cases}p(x) & \text { se } x \in X_{i} \\ 0 & \text { se } x \notin X_{i}\end{cases}$

O vetor variável indicatriz $K_{i}(x)$ representa a probabilidade do ponto x pertencer à unidade $X_{i}$. As variáveis $Y_{a i}(x)$ e $Y_{p i}(x)$ representam, respectivamente, a acumulação e a possança na unidade $X_{i}$ no ponto $x$.

Os primeiros momentos de $Y_{a i}(x)$ e $Y_{p i}(x), m_{a i}=E\left\{Y_{a i}(x)\right\}$ e $m_{p i}=E\left\{Y_{p i}(x)\right\}, i=1,2$ representam a acumulação e a espessura relativas de cada formação geológica $X_{i}$ na área $A$. 
Para as variáveis auxiliares $Y_{a}(x)$ e $Y_{p}(x)$, podem considerar-se os seguintes variogramas, adaptados da definição de variogramas multifásicos, segundo proposta por Soares (1992):

$\gamma_{Y_{a}}(h)=\frac{1}{2} E\left\{\sum_{i=1}^{N}\left[Y_{a_{i}}(x)-Y_{a_{i}}(x+h)\right]^{2}\right\} \mathrm{e}$

$\gamma_{i p}(h)=\frac{1}{2} E\left\{\sum_{i=1}^{N}\left[Y_{p_{i}}(x)-Y_{p}(x+h)\right]^{2}\right\}$

\subsection{Estimação da acumulação $a(x)$ e da pos- sança $p(x)$ na área mineralizada $A$}

Em cada ponto $x_{0}$, da área $A$, podem definir-se os estimadores de krigagem de $Y_{a i}\left(x_{0}\right)$ e $Y_{p i}\left(x_{0}\right)$, tendo, como base, as amostras vizinhas (Isaaks \& Srivastava, 1989; Journel \& Huijbregts, 1992; Soares, 2000):

$$
\begin{aligned}
& {\left[Y_{a_{i}}\left(x_{0}\right)\right]^{k}=\sum_{\alpha} \lambda_{\alpha} Y_{a_{i}}\left(x_{\alpha}\right)} \\
& {\left[Y_{p_{i}}\left(x_{0}\right)\right]^{k}=\sum_{\beta} \lambda_{\beta} Y_{p_{i}}\left(x_{\beta}\right), \text { com } \mathrm{i}=1,2}
\end{aligned}
$$

Os ponderadores $\lambda_{\alpha}$ são calculados resolvendo o sistema de krigagem e assumindo os variogramas multifásicos descritos. Em cada fase $X_{i}, i=1$, 2, podemos estimar a proporção de cada fase $X_{1}$ e $X_{2}$ na localização $x_{0}$, com base no variograma da indicatriz de $K_{i}(x)$ :

$\left[K_{i}\left(x_{0}\right)\right]^{*}=\sum_{\alpha} \lambda_{\alpha} K_{i}\left(x_{\alpha}\right)$

Assumindo a independência entre $K_{i}(x)$ e $Y_{a i}(x)$, podem dividir-se $\left[Y_{a i}\left(x_{0}\right)\right]^{*}$ - a acumulação estimada para todas as classes - em duas componentes:

$\frac{\left[Y_{a_{1}}\left(x_{0}\right)\right]^{3}}{\left[K_{1}\left(x_{0}\right)\right]^{2}}=\left[a_{1}\left(x_{0}\right)\right]^{x}$ acumulação estimada, se $x_{0}$ pertence à fase $X_{1}$, e $\frac{\left[Y_{a_{2}}\left(x_{0}\right)\right]^{*}}{1-\left[K_{1}\left(x_{0}\right)\right]^{*}}=\left[a_{2}\left(x_{0}\right)\right]^{*}$ acumulação estimada se $x_{0}$ pertence à fase $X_{2}$.

Do mesmo modo, a possança estimada pode dividir-se nas duas componentes estimadas:

$\frac{\left[Y_{p_{1}}\left(x_{0}\right)\right]^{*}}{\left[K_{1}\left(x_{0}\right)\right]^{*}}=\left[p_{1}\left(x_{0}\right)\right]^{*}$ possança estimada se $x_{0}$ pertence à fase $X_{1}$ e $\frac{\left[Y_{p_{2}}\left(x_{0}\right)\right]^{*}}{1-\left[K_{1}\left(x_{0}\right)\right]^{*}}=\left[p_{2}\left(x_{0}\right)\right]^{*}$ possança estimada se $x_{0}$ pertence à fase $X_{2}$.
Finalmente, se $x_{0}$ pertence à fase $X_{i}$, os teores são estimados por

$\left[z\left(x_{0}\right)\right]^{*}=\frac{\left[Y_{a_{i}}\left(x_{0}\right)\right]^{*}}{\left[Y_{p_{i}}\left(x_{0}\right)\right]^{*}}$

A acumulação, a possança e os teores de cada ponto $x_{0}$ de A foram estimados considerando que a transição entre as regiões $X_{1}$ e $X_{2}$ não é suave. Para a construção dos mapas finais de teores, é necessário discriminar, simultaneamente, a morfologia das unidades geológicas $X_{1}$ e $X_{2}$ disjuntas pertencentes à área $A$.

\subsection{Classificação dos mapas estimados em mapas morfológicos nas duas unidades geológicas consideradas}

Em cada ponto $x_{u}$, de uma malha regular de $A$, são conhecidas estimativas da acumulação, possança e teor conforme o ponto $x_{u}$ pertença a cada uma das unidades consideradas. Se, por exemplo, o ponto $x_{u}$ for classificado na unidade $X_{1}$, então a possança, a acumulação e o teor serão $p_{1}\left(x_{u}\right), a_{1}\left(x_{u}\right)$ e $z_{1}\left(x_{u}\right)$; se fossem classificados na unidade $X_{2}$, então a possança, a acumulação e o teor seriam dados por $p_{2}\left(x_{u}\right), a_{2}\left(x_{u}\right)$ e $z_{2}\left(x_{u}\right)$ Assim, a questão que subsiste é a classificação ou alocação de todos os blocos estimados $x_{u}$ a uma das fases $X_{1}$ ou $X_{2}$.

A transformação dos mapas estimados de acumulação e de possança em mapas morfológicos tem como base o critério da quantidade de metal estimada para cada fase $X_{i}$ : (onde $M$ é o número total de blocos estimados).

$$
a_{1}^{*}=\sum_{u=1}^{M}\left[Y_{a_{1}}\left(x_{u}\right)\right]^{*} \text { e } a_{2}^{*}=\sum_{u=1}^{M}\left[Y_{a_{2}}\left(x_{u}\right)\right]^{*}
$$

Na prática, são escolhidos os $M_{1}$ pontos (com $M=M_{1}+$ $M_{2}$ ) com os valores de acumulação relativa mais elevados, de modo que a quantidade de minério, na unidade geológica $X_{i}$, seja igual a:

$$
a_{1}=\sum_{u=1}^{M}\left[Y_{a_{1}}\left(x_{u}\right)\right]^{*}=\sum_{u=1}^{M_{1}}\left[a\left(x_{u}\right)\right]^{*} \cdot 1+\sum_{u=1}^{M_{2}}\left[a\left(x_{u}\right)\right]^{*} .0
$$

No presente caso de estudo, e dado o significado das fases $X_{1}$ e $X_{2}$, o critério utilizado é o do peso em diamantes na fase $X_{1}$. 
Delimitação e avaliação de reservas em jazigos aluvionares diamantíferos a partir da acumulação de teores

3. Exemplo de avaliação de reservas para um jazigo aluvionar de diamantes

A metodologia apresentada na seção anterior é ilustrada com um exemplo de um jazigo aluvionar de diamantes. Nesse tipo de jazigos aluvionares, as pedras tendem a aparecer concentradas em áreas, as quais recebem o nome de armadilhas (trapsites). Isto significa que se está perante uma situação em que os teores são diferenciados no espaço em regiões que evidenciam transições bruscas de teores entre os zeros e os não zeros.

O objetivo desse estudo é a caracterização da localização espacial dessas áreas de deposição preferencial das pedras de diamante, a diferenciação das duas unidades $X_{1}$ e $X_{2}$ e a estimação dos teores de acordo com um controle morfológico zonal - transições bruscas de teores entre as unidades geológicas.

As dimensões e o peso total das pedras são medidos a partir de furos verticais com diâmetro constante e possança variável. As duas formações $X_{1}$ e $X_{2}$ representam, respectivamente, as áreas com teores (quilates $/ \mathrm{m}^{3}$ ) diferentes e iguais a zero.
Na Tabela 1, apresentam-se os estatísticos básicos das variáveis estudadas amostradas em 252 sondagens (Figura 1).

As sondagens foram efetuadas numa série de perfis perpendiculares a um rio. Em cada sondagem foi amostrada a espessura de cobertura (estéril), a espessura de cascalho (unidade potencialmente diamantífera) e o peso em diamantes ou teor (quilates $/ \mathrm{m}^{3}$ ).

Para as variáveis alvo do estudo geoestatístico $K(x), Y_{a}(x)$ e $Y_{p}(x)$, foram calculados variogramas experimentais e ajustados modelos teóricos que se sintetizam na Tabela 2. Para completar o estudo, adicionou-se a variável espessura de cobertura subjacente $[e(x)]$, que não condiciona a diferenciação morfológica.

A título meramente ilustrativo, na Figura 2, mostram-se os variogramas experimentais e os modelos teóricos ajustados da acumulação e possança relativas.

Foram estimados, para a área em estudo, por krigagem, mapas para as quatro variáveis listadas na Tabela $1 . \mathrm{Na}$ Figura 3, mostram-se os mapas estimados das variáveis espessura de cobertura $e(x), K_{1}(x)$ ou probabilidade de pertença à fase $X_{1}$ (mineralizada), possança relativa $Y_{p}(x)$ e acumulação relativa $Y_{a}(x)$.
A partir da variável estimada $Y_{a 1}(x)$, acumulação relativa da fase $X_{1}$, foram derivadas imagens da acumulação para $X_{1}$, possança também para $X_{1}$, binário das fases $X_{1}$ e $X_{2}$ e teores com a metodologia explanada na seção 2.3. Os resultados são apresentados na Figura 4.

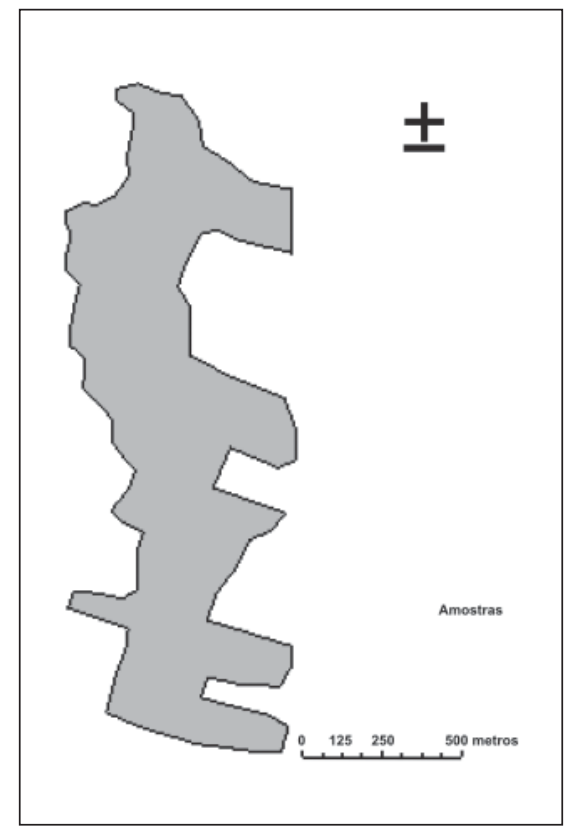

Figura 1 - Área transversalmente amostrada ao longo de um rio e localização das sondagens.

Tabela 1 - Estatísticos básicos das variáveis estudadas.

\begin{tabular}{|c|c|c|c|c|c|c|c|}
\hline & Unidades & Mínimo & Máximo & Média & Desvio-padrão & $\begin{array}{l}\text { Coeficiente } \\
\text { assimetria }\end{array}$ & Outliers \\
\hline $\begin{array}{c}\text { Espessura } \\
\text { cobertura, } e(x)\end{array}$ & $\mathrm{m}$ & 0,00 & 8,03 & 2,66 & 1,37 & 0,51 & $\begin{array}{l}\text { Direita, } 1^{\mathrm{a}} \mathrm{e} \\
2^{\mathrm{a}} \text { ordem }\end{array}$ \\
\hline $\begin{array}{c}\text { Possança } \\
\text { cascalho, } p(x)\end{array}$ & $\mathrm{m}$ & 0,00 & 5,56 & 1,10 & 0,97 & 2,08 & $\begin{array}{c}\text { Direita, } 1^{\mathrm{a}} \mathrm{e} \\
2^{\mathrm{a}} \text { ordem }\end{array}$ \\
\hline Teor $z(x)$ & Quilates $/ \mathrm{m}^{3}$ & 0,00 & 2,59 & 0,10 & 0,26 & 5,12 & $\begin{array}{l}\text { Direita, } 1^{\mathrm{a}} \mathrm{e} \\
2^{\mathrm{a}} \text { ordem }\end{array}$ \\
\hline $\begin{array}{c}\text { Acumulação } \\
a(x)\end{array}$ & Quilates $/ \mathrm{m}^{2}$ & 0,00 & 1,52 & 0,07 & 0,18 & 4,09 & $\begin{array}{c}\text { Direita, } 1^{\mathrm{a}} \mathrm{e} \\
2^{\mathrm{a}} \text { ordem }\end{array}$ \\
\hline Binário $K_{1}(x)$ & & 0,00 & 1,00 & 0,35 & ---- & ----- & ----- \\
\hline
\end{tabular}


José A. Almeida

Tabela 2 - Parâmetros dos modelos teóricos ajustados aos variogramas experimentais.

\begin{tabular}{c|c|c|c|c}
\hline \multirow{2}{*}{ Variável } & \multirow{2}{*}{$\boldsymbol{c}_{\boldsymbol{0}}$} & \multicolumn{3}{|c}{ Modelo teórico } \\
\cline { 3 - 5 } & & Tipo de função & $\boldsymbol{c}_{\mathbf{1}}$ & $\boldsymbol{a}_{\mathbf{1}}$ \\
\hline Espessura de cobertura $e(x)$ & 0,0 & Esférico, isótropo & 1,86 & $350 \mathrm{~m}$ \\
\hline Variável morfológica binária $k(x)$ & 0,0 & Exponencial, isótropo & 0,23 & $175 \mathrm{~m}$ \\
\hline Acumulação relativa $Y_{a}(x)$ & 0,0 & Exponencial, isótropo & 0,03 & $175 \mathrm{~m}$ \\
\hline Possança relativa $Y_{p}(x)$ & 0,0 & Exponencial, isótropo & 0,60 & $175 \mathrm{~m}$ \\
\hline
\end{tabular}

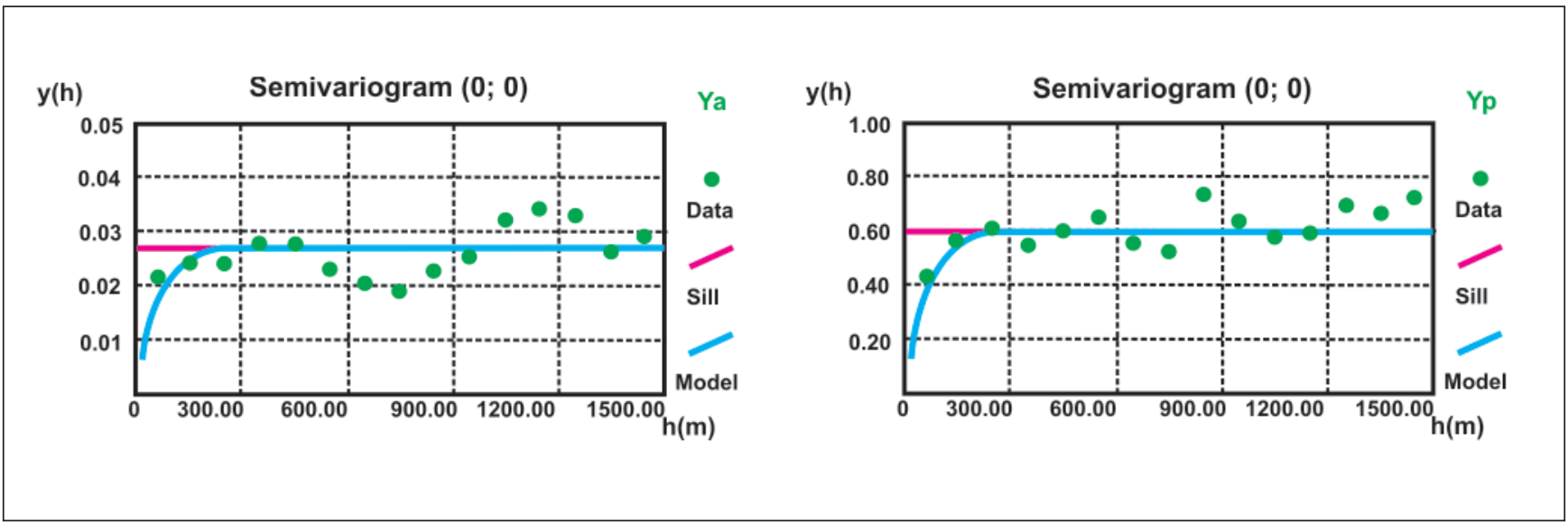

Figura 2 - Variogramas experimentais e modelos teóricos das variáveis acumulação e possança relativas.
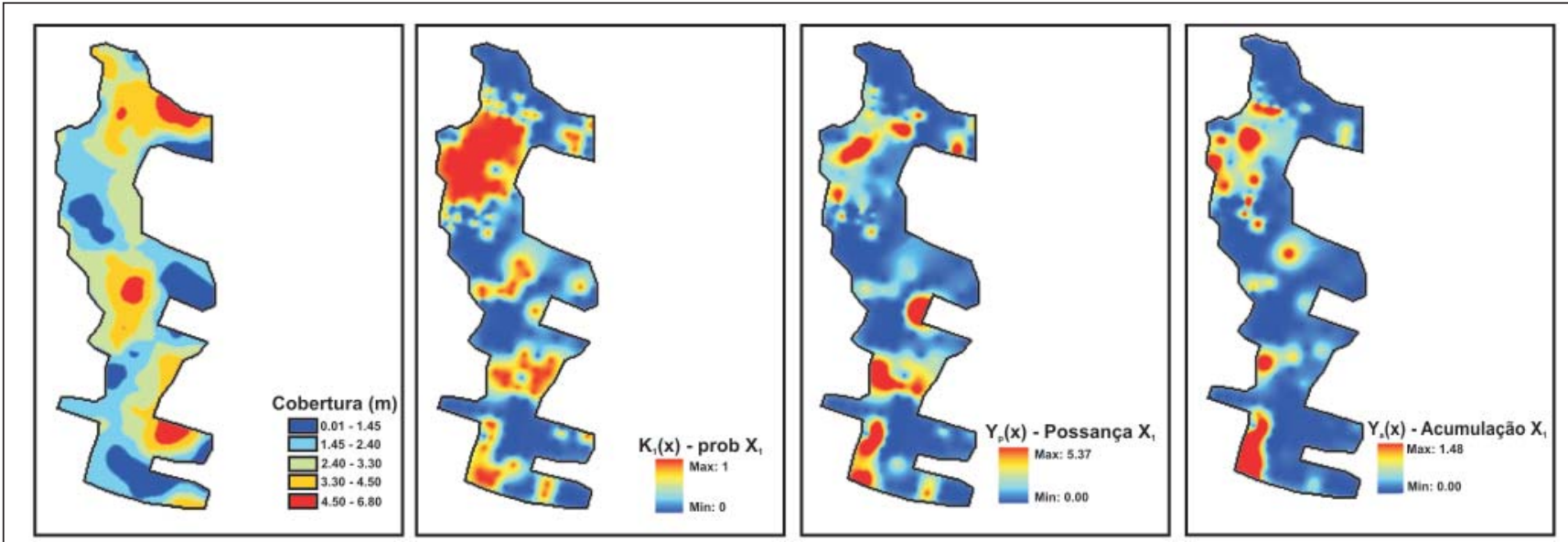

Figura 3 - Mapas estimados das variáveis $e(x), K_{1}(x), Y_{p}(x)$ e $Y_{a}(x)$.

Se se compararem os valoreslimites representados nas imagens com os dos dados experimentais da Tabela 1 , observa-se que as leis de distribuição dos valores estimados são coerentes com o que se pode esperar de uma estimação por krigagem normal, ou seja, tais valores são ligeiramente atenuados face aos valores amostrados.

\section{Considerações finais}

Na avaliação de reservas de depósitos minerais evidenciando elevada descontinuidade nas propriedades internas - teores, porosidade, classes de permeabilidade -, a caracterização espacial dessas propriedades deve de ter em conta o efeito zonal provocado pelas transições morfológicas.

A metodologia apresentada te, como base, a estimação conjunta da forma das diferentes unidades e dos teores de uma propriedade que é discriminada pela 

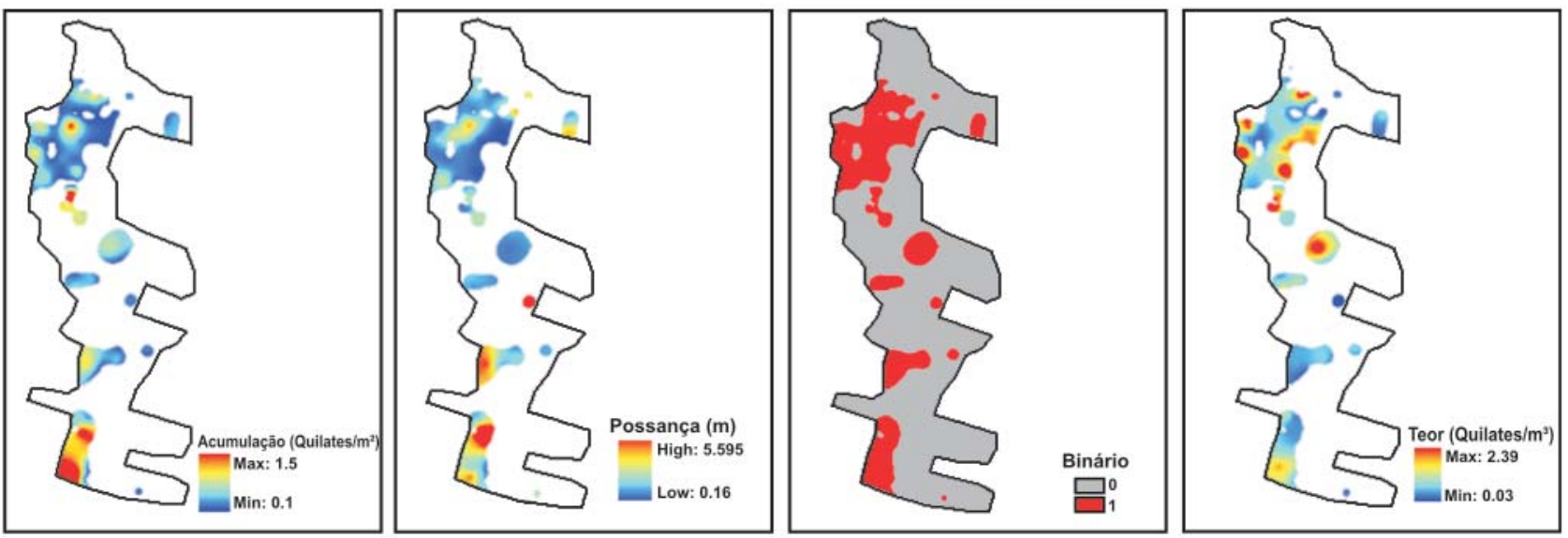

Figura 4 - Imagens obtidas para a acumulação, a possança, as fases $X / X$ e o teor.

morfologia. Uma vez que os teores são perfeitamente discriminados pela morfologia, esta também pode ser discriminada pelos teores, que são interpolados em cada unidade. O exemplo de avaliação de reservas de um jazigo aluvionar diamantífero, em que a distribuição de teores tem uma zonalidade espacial bem definida, mostra a aplicabilidade da metodologia proposta.

A alternativa seria a estimação da morfologia, seguida da estimação condicional dos teores. Contudo, num exemplo como o que foi utilizado nesse trabalho, podem ser apontadas duas grandes desvantagens: i) a presença de amostras isoladas; ii) a fase mineralizada constituir pouco mais de 30\% das amostras, o que dificultaria - e muito - a modelação de variogramas das variáveis acumulação e possança condicionais.

\section{Referências bibliográficas}

ALMEIDA, J., SOARES, A. O., ALBUQUERQUE, A. Zonal control to estimate classes of histograms: application to a diamond alluvial deposit. In: BAAFI, E.Y., SCHOFIELD, N.A. (ed.) Geostatistics Wollongong'96, Kluwer Academic Pub., Dordrecht, 2: p. 658-669, 1997.

CHILES, J. P., DELFINER, P. Geostatistics: modeling spatial uncertainty. New York: Wiley, 1999. 720p.
GARCIA-PEREIRA, H., Cálculo geoestatístico de reservas em economia mineira. Geosistemas, n. 3, p. 89-93, 1994.

GOOVAERTS, P. Geostatistics for natural resources characterization. New York: Oxford University Press, 1997. 483p.

ISAAKS, E. H., SRIVASTAVA, R. M. An introduction to applied geostatistics. New York: Oxford University Press, 1989. 561p.

JOURNEL, A. G., ISAAKS, E. Conditional indicator simulation: application to a saskatchewan uranium deposit. Mathematical Geol., 16, p. 685-718, 1984.

JOURNEL, A. G., HUIJBREGTS, C. J. Mining geostatistics. New York: Academic Press, 1992. 600p.

MARSHALLA, T. R., BAXTER-BROWNB, R. Journal of Geochemical Exploration, v. 53, Issues 1-3, p. 277-292, 1995.

MATHERON, G. La Théorie des Variables Regionalisées et ses Applications. Fontainebleau: Ecole des Mines de Paris, 1970. (Les Cahiers du Centre de Morphologie Mathematique. Fascicule $5)$.

ROMBOUTS, L. Sampling and statistical evaluation of diamond deposits. Journal Geochemical Exploration, 53, p. 351-367, 1995.

SOARES, A. O. Geostatistical estimation of orebody geometry: morphological kriging. Mathematical Geol., 22, p. 787-802, 1990.

SOARES, A. O. Geostatistical estimation of multiphase structures. Mathematical Geol., 24, p. 149-160, 1992.

SOARES, A. O. Geoestatística para as ciências da Terra e do ambiente. Coleção Ensino da Ciência e da Tecnologia. Lisboa: IST Press, 2000. 206 p.

Artigo recebido em 09/03/2009 e aprovado em 21/05/2009.

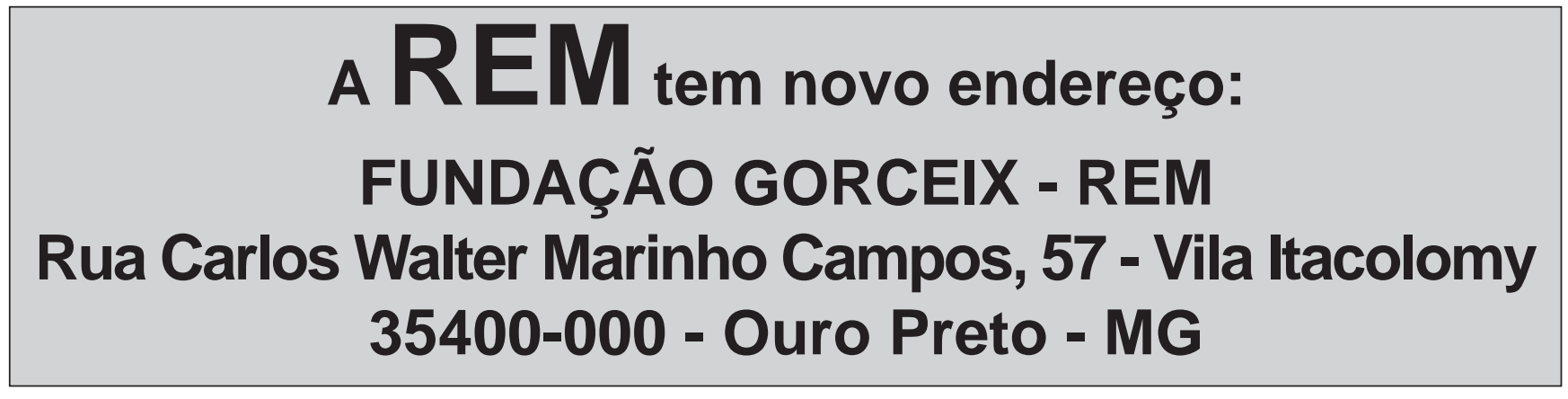

